\title{
Texturing in Earth's inner core due to preferential growth in its equatorial belt
}

\author{
Renaud Deguen ${ }^{\mathrm{a}, \mathrm{b}}$, Philippe Cardin ${ }^{\mathrm{a}, *}$, Sébastien Merkel ${ }^{\mathrm{c}}$, Ricardo A. \\ Lebensohn $^{\mathrm{d}}$ \\ ${ }^{a}$ Institut des Sciences de la Terre (ISTerre), CNRS, Université Joseph-Fourier Grenoble \\ 1, Grenoble, France. \\ ${ }^{b}$ Earth and Planetary Sciences, Johns Hopkins University, Baltimore, Maryland, USA. \\ ${ }^{c}$ Unité Matériaux et Transformations (UMET), CNRS, Université Lille 1, Villeneuve \\ d'Ascq, France. \\ ${ }^{d}$ Materials Science and Technology Division, Los Alamos National Laboratory, Los \\ Alamos, New Mexico, USA.
}

\section{Abstract}

We propose an extension of the model by Yoshida et al. (1996), where deformation in the inner core is forced by preferential growth in the equatorial belt, by taking into account the presence of a stable compositional stratification. Stratification inhibits vertical motion, imposes a flow parallel to isodensity surfaces, and concentrates most deformation in a shallow shear layer of thickness $\sim B^{-1 / 5}$, where $B$ is the dimensionless buoyancy number. The localization of the flow results in large strain rates and enables the development of a strong alignment of iron crystals in the upper inner core. We couple our dynamical model with a numerical model of texture development and compute the time evolution of the lattice preferred orientation of different samples in the inner core. With sufficient stratification, texturing is significant in the uppermost inner core. In contrast, the deeper inner core

\footnotetext{
${ }^{*}$ Corresponding author

Email address: philippe.cardin@ujf-grenoble.fr (Philippe Cardin )
} 
is unaffected by any flow and may preserve a fossil texture. We investigate the effect of an initial texture resulting from solidification texturing at the ICB. In the present inner core, the deformation rate in the shallow shear layer is large and can significantly alter the solidification texturing, but the solidification texture acquired early in the inner core history can be preserved in the deeper part. Using elastic constants from $a b$ initio calculations, we predict different maps of anisotropy in the modern inner core. A model with both solidification texturing and subsequent deformation in a stratified inner core produces a global anisotropy in reasonable agreement with seismological observations.

Keywords: inner core, anisotropy, HCP iron, texturation, crystallization

\section{Introduction}

The inner core of the Earth exhibits a noticeable anisotropy in P-wave velocity and attenuation (Poupinet et al., 1983; Morelli et al., 1986; Woodhouse et al., 1986; Souriau, 2007), which may reflect structural and dynamical complexity. The main observation is a $\sim 3 \%$ anisotropy with cylindrical symmetry, with the fast axis aligned with the axis of rotation of the Earth or possibly slightly tilted (Su et al., 1996). The degree of inner core anisotropy is increasing with depth (Souriau, 2003), with no strong anisotropy in the upper 150-200 km (Song and Helmberger, 1995), and perhaps still different [but poorly constrained (Calvet et al., 2006)] seismic properties in an innermost inner core (Ishii and Dziewoński, 2002; Niu and Chen, 2008). There is also a growing consensus on the presence of an hemispherical asymmetry in the seismic properties of the upper part of the inner core (Tanaka and 
Hamaguchi, 1997). An anisotropy in inner core seismic attenuation is also observed (Souriau and Romanowicz, 1996, 1997; Yu and Wen, 2006a) and shows hemispherical variations (Garcia, 2002; Yu and Wen, 2006b) which are reminiscent of the pattern of the anisotropy.

The seismic anisotropy of the inner core is most often interpreted as resulting from the lattice preferred orientation (LPO) of elastically anisotropic iron crystals. Processes for creating iron LPO in the inner core include solidification at the inner core boundary (ICB) (Karato, 1993; Bergman, 1997; Brito et al., 2002), plastic deformation (Jeanloz and Wenk, 1988; Karato, 1999; Wenk et al., 2000a; Buffett and Wenk, 2001), or stress-induced recrystallization (Yoshida et al., 1996). It is not, however, unlikely that a combination of several mechanisms are - or have been - active. To what extent solidification texturing can be reworked by subsequent deformation might well be a critical point to understand the observed radial variations in anisotropy and attenuation.

A number of deformation models have been proposed, including thermal convection (Jeanloz and Wenk, 1988; Wenk et al., 1988, 2000a; Weber and Machetel, 1992; Buffett, 2009), continuous deformation forced by aspherical growth (Yoshida et al., 1996), and flow induced by the outer core magnetic field (Karato, 1999; Buffett and Wenk, 2001; Takehiro, 2010). The viability of most of these mechanisms depends on the thermal and chemical state of the inner core. Apart from the mechanisms proposed by Buffett and Wenk (2001) and Takehiro (2010), all involve a large radial flow (Jeanloz and Wenk, 1988; Yoshida et al., 1996; Karato, 1999; Wenk et al., 2000a) and will be inhibited by a stable stratification in the inner core (Buffett 
and Bloxham, 2000; Deguen and Cardin, 2009). The 'convective translation' proposed recently by Monnereau et al. (2010) and Alboussière et al. (2010) also requires an unstable stratification.

In this paper, we extend the model proposed by Yoshida et al. (1996) to include the effect of a stable density stratification in the inner core. In section 2 , we discuss the thermal and compositional state of the inner core before introducing our dynamical model in section 3 . We then concentrate our efforts on connecting our geodynamic model with simulations of LPO development in mineral aggregates using the viscoplastic self-consistent (VPSC) model of Lebensohn and Tomé (1993). The mineralogical model we used is discussed in section 4. From our numerical models of inner core deformation, we track the deformation history of markers inside the inner core and calculate the evolution of their LPO (section 5). The results are used in section 6 to calculate the resulting $\mathrm{P}$-wave velocity anisotropy.

\section{Dynamical model}

\subsection{Thermal state of the inner core}

The thermal state of the inner core results from a competition between cooling at the ICB and extraction of the inner core internal heat. The inner core would be stably stratified (subadiabatic) if its growth is slow enough to allow thermal conduction to extract most of its internal heat. In contrast, the inner core would develop an unstable thermal stratification, and may be able to convect, if its growth has been fast enough. The thermal evolution of the inner core has been investigated in some details elsewhere (Sumita et al., 1995; Yukutake, 1998; Buffett, 2009; Deguen and Cardin, 2009, 2011) but, 
unfortunately, uncertainties on most important parameters (growth rate of the inner core, thermal diffusivity, adiabat and Clapeyron slopes) are such that it is not possible at present to conclude about the thermal stratification of the inner core. Deguen and Cardin (2011) estimate that the inner core would have developed a stable thermal stratification if its age is larger than $0.9 \pm 0.6$ Gy. This fully overlaps with inner core age estimates from core thermal evolution models, e.g. $1 \pm 0.5$ Gy in Labrosse et al. (2001) and $1.15 \pm 0.75$ Gy in Nimmo (2007), which implies that whether the inner core is subadiabatic or superadiabatic is not known. Here, we focus on the case where the inner core has a stable subadiabatic thermal stratification, keeping in mind that this is one of two equally likely possibilities.

\subsection{Chemical stratification in the inner core}

Additional density stratification can come from compositional stratification in the inner core. Light elements present in the core partition upon freezing, which implies that the composition of the outer core evolves in response to inner core solidification. Since the composition of the newly crystallized solid at the ICB is linked with the outer core composition by partition coefficients, the temporal evolution of the composition of the outer core results in a compositional stratification of the inner core. Gradual enrichment of the outer core in light incompatible elements can result in a stable chemical stratification in the inner core.

We assume here that the outer core is well-mixed, has negligible chemical interactions with the mantle, and that the partition coefficients are constant. Chemical diffusion being negligible in the inner core, the evolution of the concentration of the light element $c^{\ell}(t)$ in the liquid outer core is given by 
the Rayleigh distillation law (e.g. Albarède, 1996),

$$
c^{\ell}(t)=c_{0}^{\ell}\left[1-\frac{M_{i c}(t)}{M_{0}}\right]^{D-1},
$$

where $c_{0}^{\ell}$ is the concentration in the core prior to inner core nucleation, $D$ is the partition coefficient (the ratio of the concentration in the solid to that in the liquid), $M_{i c}$ the mass of the inner core, and $M_{0}$ the mass of the core. For simplicity, we approximate $M_{i c}(t) / M_{0} \approx\left(r_{i c b}(t) / r_{c}\right)^{3}$. The light element concentration $c$ in the solid at the ICB at time $t$ is given by $D c^{\ell}(t)$, which implies a compositional profile in the inner core given by

$$
c(r)=D c_{0}^{\ell}\left(1-\left(\frac{r}{r_{c}}\right)^{3}\right)^{D-1} .
$$

The resulting radial density variations profile is

$$
\begin{aligned}
\Delta \rho_{c}(r)=\alpha_{c} \rho[c(r)-c(0)] & =\alpha_{c} \rho D c_{0}^{\ell}\left[\left(1-\left(\frac{r}{r_{c}}\right)^{3}\right)^{D-1}-1\right] \\
& \approx \alpha_{c} \rho D(1-D) c_{0}^{\ell}\left(\frac{r}{r_{c}}\right)^{3}
\end{aligned}
$$

where $\rho$ is the mean density in the inner core and $\alpha_{c}=(1 / \rho)(\partial \rho / \partial c)$ is the compositional expansion coefficient. Equation (4) shows that the chemical stratification is approximately proportional to $D(1-D)$ : stratification would be maximum if $D=0.5$, and is small if the distribution coefficient is either small or close to one.

The magnitude of the stratification depends on the nature and abundance of the light elements present in the core. Recent models favour O, Si and $\mathrm{S}$ as the most plausible alloying elements. Ab initio calculations of the partitioning behaviour of $\mathrm{O}$, Si and S by Alfè et al. (2002) suggest that Si 
and $\mathrm{S}$ both partition weakly (with similar partition coefficients, $D^{\mathrm{Si}, \mathrm{S}}=0.8$ ) while, in contrast, $\mathrm{O}$ partitions strongly, $D^{\mathrm{O}}=0.02$ (values of $D$ are converted from molar ratios to mass ratios). Alfè et al. (2002) estimate that the outer core contains 5.6 wt. \% of $\mathrm{Si}$ and/or $\mathrm{S}$ and 2.5 wt. \% of $\mathrm{O}$, in good agreement with the geochemical model of Allègre et al. (1995), and that the inner core contains 4.4 wt. \% of $\mathrm{Si} / \mathrm{S}$ and negligible amount of O. Badro et al. (2007) used additional constraints from experimentally measured compressional wave velocity of $\mathrm{Fe}-\mathrm{Si}$, Fe-S and Fe-O alloys. In their model, $\mathrm{S}$ is unlikely to be a major component of the inner core, the outer core contains 2.8 wt. \% $\mathrm{Si}$ and 5.3 wt. \% O, and the inner core is constituted of 2.3 wt. $\%$ Si and 0.1 wt. \% O.

With the distribution coefficients calculated by Alfè et al. (2002), the chemical models discussed above, and $\alpha_{c} \simeq-1$ [see Deguen and Cardin (2011)], we find that the density stratification associated with $\mathrm{Si} / \mathrm{S}$ is much larger than that associated with $\mathrm{O}$. We therefore model the inner core as a Fe- $(\mathrm{Si}, \mathrm{S})$ binary mixture with a distribution coefficient equal to 0.8 . The difference in density across the inner core is $\Delta \rho_{c} \sim-2$ to $-5 \mathrm{~kg} \mathrm{~m}^{-3}$. This is probably an upper bound because departures from the assumptions behind this derivation would result in a weaker stratification. As noted by Alboussière et al. (2010), variations of the effective partition coefficient resulting from changes in the efficiency of melt extraction by compaction (Sumita et al., 1996) and interdendritic convection (Loper, 1983; Worster, 1997) in a mushy layer at the ICB may significantly decrease the magnitude of the chemical stratification. 


\subsection{Dynamic equations for a stratified inner core}

We build a model for a thermally and chemically stratified inner core according to the two previous subsections. Under these conditions, the inner core stays at rest except if external forcings, such as pressure forcings (Yoshida et al., 1996) or magnetic forcings (Karato, 1999; Buffett and Bloxham, 2000; Buffett and Wenk, 2001), are imposed. Here, we follow the ideas of Yoshida et al. (1996) and focus on how differential growth of the inner core generates motion in a stratified inner core. For simplicity, we only consider the chemical contribution to the density stratification.

\subsubsection{Equatorial growth of the inner core}

The growth of the inner core is primarily due to its cooling by action of convective motions in the liquid outer core, also responsible for the geodynamo generation. The predominance of the axial geomagnetic dipole throughout the Earth's history demonstrates the permanent key role of the Coriolis force in the fluid motion in the outer core. Thermal convection in the outer core is made of columnar vortices aligned with the axis of rotation. This quasi geostrophic flow, first predicted by Busse (1970) and later confirmed by numerical or experimental means (Zhang, 1992; Dormy et al., 2004; Cardin and Olson, 1994) has a strong influence on convective heat transfer (Aubert et al., 2008; Aurnou et al., 2008).

Accordingly, it is expected that heat is extracted more efficiently from the equatorial band than from the polar regions of the inner core, which results in faster crystallization in the equatorial band. Following Yoshida et al. (1996), we model this by introducing a spherical harmonic degree 2 dependence in 
the rate of crystallization of the inner core $u$,

$$
u(\theta, t)=u_{i c b}(t)\left(1-S_{2} \frac{3 \cos ^{2} \theta-1}{2}\right)
$$

where $\theta$ is the colatitude and $S_{2}$ is a dimensionless parameter that measures the differential growth. In calculations by Aubert et al. (2008), the crystallization rate at the equator is approximately twice larger than that at the poles, which is equivalent to $S_{2}=2 / 5$.

Preferential growth of the inner core in the equatorial belt produces an out-of-equilibrium topography, which, if not sustained by heterogeneous solidification, would relax toward hydrostatic equilibrium. The problem is very similar to that of post-glacial rebound, and the timescale of viscous relaxation $\tau_{\eta}$ can be calculated by extending to spherical geometry the classical calculation of topography relaxation in a semi-infinite domain (e.g. Turcotte and Schubert, 2002). We find

$$
\tau_{\eta}=\frac{19}{5} \frac{\eta}{\Delta \rho_{\mathrm{icb}} g r_{\mathrm{icb}}} \simeq\left(\frac{\eta}{10^{18} \text { Pa.s }}\right)\left(\frac{1221 \mathrm{~km}}{r_{\mathrm{icb}}}\right)^{2} \times 40 \text { year }
$$

where $\eta$ is the dynamic viscosity of iron in the inner core, and $g$ the acceleration of gravity. Since the relaxation timescale is very small in comparison to the timescale of boundary conditions evolution and inner core growth, the inner core topography must be in a quasi-steady state, with the growth rate anomaly balanced by the continuous relaxation of the ICB topography (Yoshida et al., 1996). The radial velocity at the ICB is therefore prescribed to be equal to the opposite of the anomalous solidification rate.

This gives boundary conditions for the radial velocity; boundary conditions for the horizontal velocity are given by the assumption that the ICB is a shear stress free boundary. 
The newly crystallized material has a solute concentration $c\left(r_{i c b}(t)\right)$ given by $(2)$.

\subsubsection{Equations of motion in a growing inner core}

We consider an incompressible fluid of constant viscosity $\eta$ in a spherical domain $\left(r<r_{i c b}\right)$. The Boussinesq approximation is valid and the momentum equation is written as

$$
\mathbf{0}=-\boldsymbol{\nabla} p+\alpha_{c} \bar{\rho} c \mathbf{g}+\eta \boldsymbol{\nabla}^{2} \mathbf{v}
$$

where $p$ is the dynamic pressure, $\alpha_{c} c=(\rho-\bar{\rho}) / \bar{\rho}$ is the density perturbation from the static density field. Inertial terms have been neglected as the Reynolds number of the flow is vanishingly small. Taking the curl of the Stokes equation (7) gives

$$
\mathbf{0}=-\frac{\alpha_{c} \bar{\rho} g^{\prime}}{\eta} \frac{\partial c}{\partial \theta} \mathbf{e}_{\phi}+\nabla^{2} \boldsymbol{\nabla} \times \mathbf{v}
$$

where $g$ has been assumed to be linear in radius, and $g^{\prime}=d g / d r$. Taking advantage of the incompressibility of the velocity field, we can introduce the poloidal $(P)$ and the toroidal $(T)$ scalars to describe the velocity field $\mathbf{v}=\boldsymbol{\nabla} \times(T \mathbf{r})+\boldsymbol{\nabla} \times \boldsymbol{\nabla} \times(P \mathbf{r})$ which are projected onto the basis of the axisymmetric spherical harmonics $(P, T)=\sum\left(P_{l}(r), T_{l}(r)\right) Y_{l}^{0}(\theta)$. Taking $\mathbf{e}_{\mathbf{r}} \cdot \boldsymbol{\nabla} \times(8)$ and $\mathbf{e}_{\mathbf{r}} \cdot \boldsymbol{\nabla} \times \boldsymbol{\nabla} \times(8)$, we find

$$
D_{l}^{2} P_{l}=\frac{\alpha_{c} \bar{\rho} g^{\prime} r}{\eta} c_{l} \quad \text { and } \quad D_{l}^{2} T_{l}=0,
$$

where $c_{l}$ is the degree $l$ spherical harmonic component of the solute concentration field and $D_{l}$ is the Laplacian operator defined by $\frac{\partial^{2}}{\partial r^{2}}+\frac{2}{r} \frac{\partial}{\partial r}-\frac{l(l+1)}{r^{2}}$.

At the ICB, we deduce from (9) and (5) that the toroidal flow is zero and that, consequently, the velocity field is fully described by its poloidal part. To 
write (9) in a dimensionless form, we scale the length by $r_{i c b}(t)$, the velocity by $u_{i c b}(t)$ and the solute concentration by the difference of concentration $\Delta c(t)=c\left(r_{i c b}\right)-c(0)$ between the concentration at the top of the growing inner core and the initial concentration at the center. The poloidal part of (9) becomes

$$
D_{l}^{2} \tilde{P}_{l}=B \tilde{c}_{l},
$$

where ${ }^{\sim}$ stands for the dimensionless variables and $B$, the dimensionless buoyancy number, is defined as

$$
B=\frac{\alpha_{c} \bar{\rho} g^{\prime} \Delta c(t) r_{i c b}^{3}(t)}{\eta u_{i c b}(t)} .
$$

$B$ increases significantly during the growth of the inner core as a result of the combined effect of increasing stratification $\left(\Delta c \propto r_{i c b}^{3}\right.$ and $\left.g \propto r_{i c b}\right)$, decreasing solidification rate, and increasing length scale $r_{i c b}$, which makes the viscous transfer of momentum less efficient. If we assume that the inner core radius increases as the square root of time [a reasonable first order approximation (Buffett et al., 1992)], $B$ evolves as $r_{i c b}^{7}$. Since $\alpha_{c}$ is negative, $B$ is negative, which corresponds to a stably stratified system. The actual value of $B$ in the inner core suffers from very large uncertainties on the solidstate viscosity of iron in those conditions (Yoshida et al., 1996; Buffett, 1997; Van Orman, 2004), with published estimates ranging from $10^{11}$ Pa.s to $10^{21}$ Pa.s. With $\Delta \rho_{c}=-5 \mathrm{~kg} \mathrm{~m}^{-3}$ and $u_{i c b}=3 \times 10^{-11} \mathrm{~m} \cdot \mathrm{s}^{-1}(1 \mathrm{~mm} / \mathrm{yr})$, the present value of $B\left(\right.$ noted $\left.B^{*}=B(\tilde{t}=1)\right)$ would be

$$
B^{*}=-10^{6} \times\left(\frac{10^{18} \text { Pa.s }}{\eta}\right) .
$$

We explore a large range of $B^{*}$ value, going from $B^{*}=0$ [no stratification, Yoshida et al. (1996)] to $B^{*}=-10^{9}$. 


\subsubsection{Light element transport equation}

The solute transport equation is written as

$$
\frac{\partial c}{\partial t}+\mathbf{v} \cdot \nabla c=0
$$

Taking into account the inner core secular growth is done naturally with our choice of length scale which is the time dependent radius of the inner core $r_{i c b}(t)$. The radial domain is defined by $\tilde{r}=r / r_{i c b}(t) \in[0,1]$ with a fixed boundary at $\tilde{r}=1$. The time derivative in the new coordinate system $\tilde{\mathcal{R}}$ is written (Crank, 1984) as

$$
\left.\frac{\partial}{\partial t}\right|_{\tilde{\mathcal{R}}}=\left.\frac{\partial}{\partial t}\right|_{\mathcal{R}}-\left.\left.\frac{\partial \tilde{r}}{\partial t}\right|_{\mathcal{R}} \frac{\partial}{\partial \tilde{r}}\right|_{t}=\left.\frac{\partial}{\partial t}\right|_{\mathcal{R}}+\left.\tilde{r} \frac{u_{i c b}}{r_{i c b}} \frac{\partial}{\partial \tilde{r}}\right|_{t} .
$$

This allows the equation of solute transport to be written in the new referential as

$$
\frac{\partial \tilde{c}}{\partial \tilde{t}}=\frac{u_{i c b} \tau_{i c}}{r_{i c b}}\left(\tilde{r} \tilde{\mathbf{e}_{\mathbf{r}}}-\tilde{\mathbf{v}}\right) \cdot \tilde{\nabla} \tilde{c}-\frac{\dot{\Delta} c \tau_{i c}}{\Delta c} \tilde{c},
$$

where time has been scaled by the age of the inner core, $\tau_{i c}$, and other variables as in section 2.3.2.

The first term in the RHS of equation (15) comes from the moving boundary transformation and corresponds to an apparent inward advection in the new reference frame. The last term in the RHS comes from the time dependence of the prescribed concentration scale $\Delta c(t)(\dot{\Delta} c$ stands for the time derivative of $\Delta c$ ). If we assume that the inner core radius grows as the square root of time, and that the compositional evolution takes the simplified form (4), then $\left(u_{i c b} \tau_{i c}\right) / r_{i c b}=1 / 2$ and $\left(\dot{\Delta c} \tau_{i c}\right) / \Delta c$ simplifies to $3 / 2$.

\subsubsection{Numerical implementation and simulations}

We implement the two equations $(10,15)$ into a meridional plane, with a spectral description for the horizontal dependence and a finite difference 
$B^{*}=0$

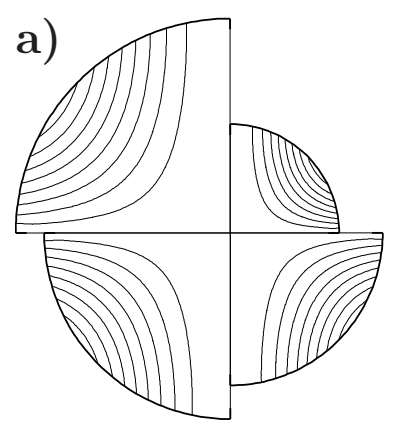

b)

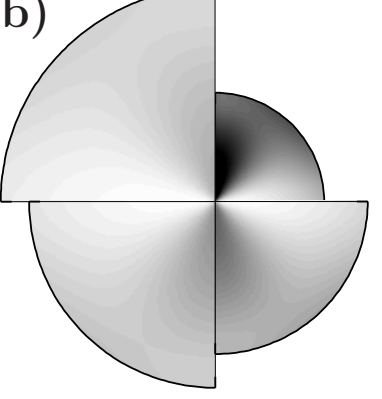

c)

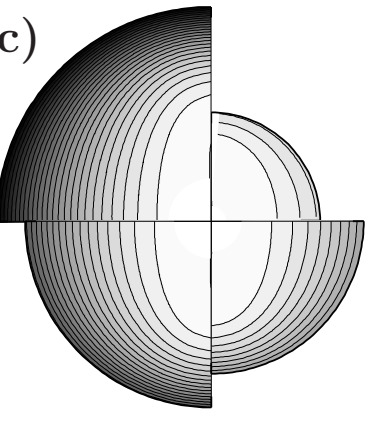

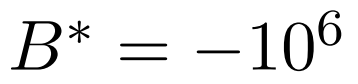

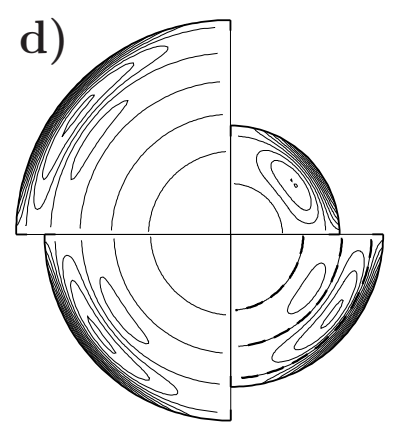

$\psi$
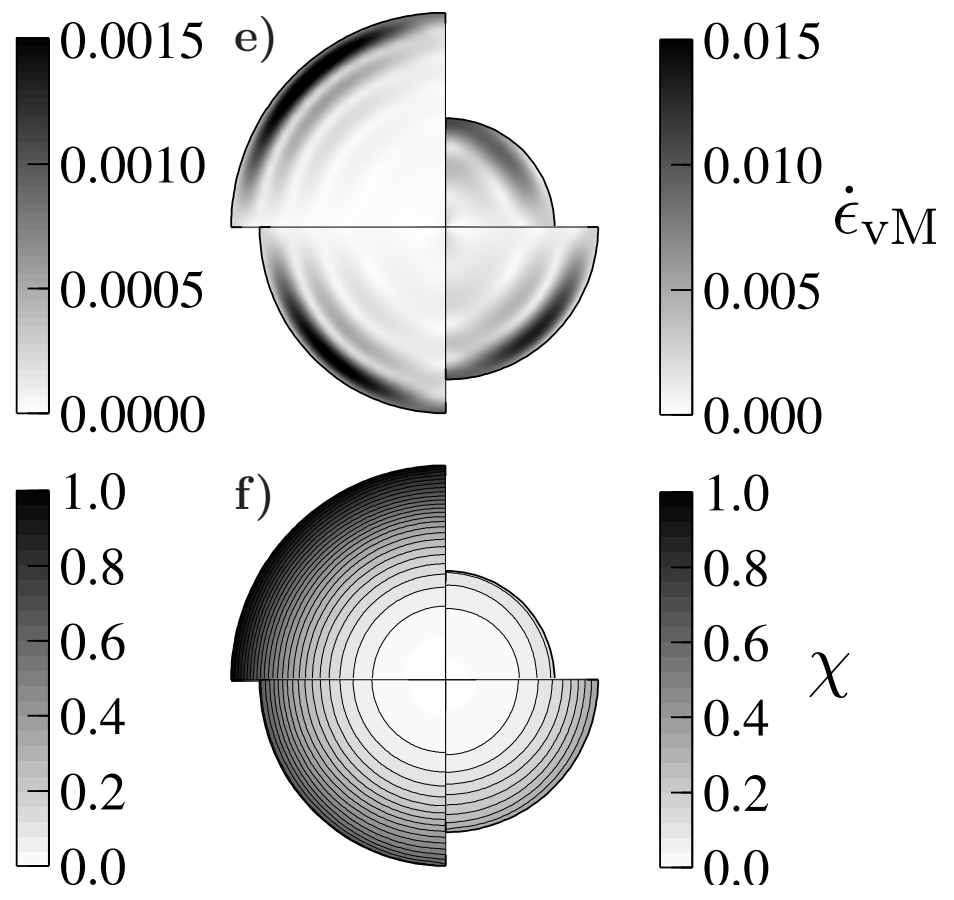

Figure 1: Time evolution of the flow induced by preferential growth of the inner core at the equator for two values of the dimensionless buoyancy number, $B^{*}=0$ (no stratification) and $B^{*}=-10^{6}$ (stratified flow). All subfigures are divided into four quadrants, which correspond to non-dimensional times $\tilde{t}=0.25,0.5,0.75$ and 1 , starting clockwise from the upper right quadrant; the radius of each quadrant reflects the value of $r_{i c b}(t)$. a) and d): Contours of the stream function $\psi$. b) and e): von Mises equivalent strain rate $\dot{\epsilon}_{\mathrm{vM}}$, in $\mathrm{Myr}^{-1}$. c) and $\left.\mathrm{f}\right)$ : Non-dimensional light-element concentration $\chi=c / \Delta c(\tilde{t}=1)$. The computations were made with $S_{2}=2 / 5$, wkich corresponds to a solidification rate two times higher at the equator than at the poles. 
scheme for the radial description. The radial mesh can be contracted in the outermost part of the domain for large $B$. Boundary conditions at $\tilde{r}=1$ are : $\forall l \neq 2, \tilde{P}_{l}=0, \tilde{P}_{2}=S_{2}$, and $\forall l, \frac{\partial^{2} \tilde{P}_{l}}{\partial \tilde{r}^{2}}=[2-l(l+1)] \tilde{P}_{l}$ (shear stress free condition), $\tilde{c_{0}}=1$ and $\forall l \neq 0, \tilde{c}_{l}=0$. The non linear term $\tilde{\mathbf{v}} \cdot \tilde{\nabla} \tilde{c}$ is evaluated in the physical space at each time step. A semi-implicit CrankNickolson scheme is implemented for the time evolution of the linear terms and an Adams-Bashforth procedure is used for the non-linear term. We typically use time steps of order $10^{-4}$.

We start the simulations with a small inner core $(\tilde{r}=0.1)$ and let evolve the system to the final time $\tilde{t}=1$. We track some fluid particles to compute their trajectories and their stress tensor $\dot{\epsilon}$ by bilinear interpolation in the physical space. These quantities are saved and used as inputs for the mineralogical model.

\section{Results of dynamical simulations}

Fig. 1 presents snapshots of the stream function $\psi$ (computed from the poloidal component of the axisymmetric velocity, $\psi=r \sin \theta \frac{\partial P}{\partial \theta}$ ), the von Mises equivalent strain rate $\dot{\epsilon}_{\mathrm{vM}}$, and the solute field. The isocontours of $\psi$ show the flow pattern. $\dot{\epsilon}_{\mathrm{vM}}$ is a measure of the deviatoric strain rate (Tomé et al., 1984; Wenk et al., 2000a), and can be used to highlight the regions of intense deformation.

\subsection{Neutral stratification, $B=0$}

The case $B=0$ (Fig. 1 left column) corresponds to no back reaction of the buoyancy force on the flow. Our numerical results are in good agreement with the analytical model derived by Yoshida et al. (1996). The absence of 

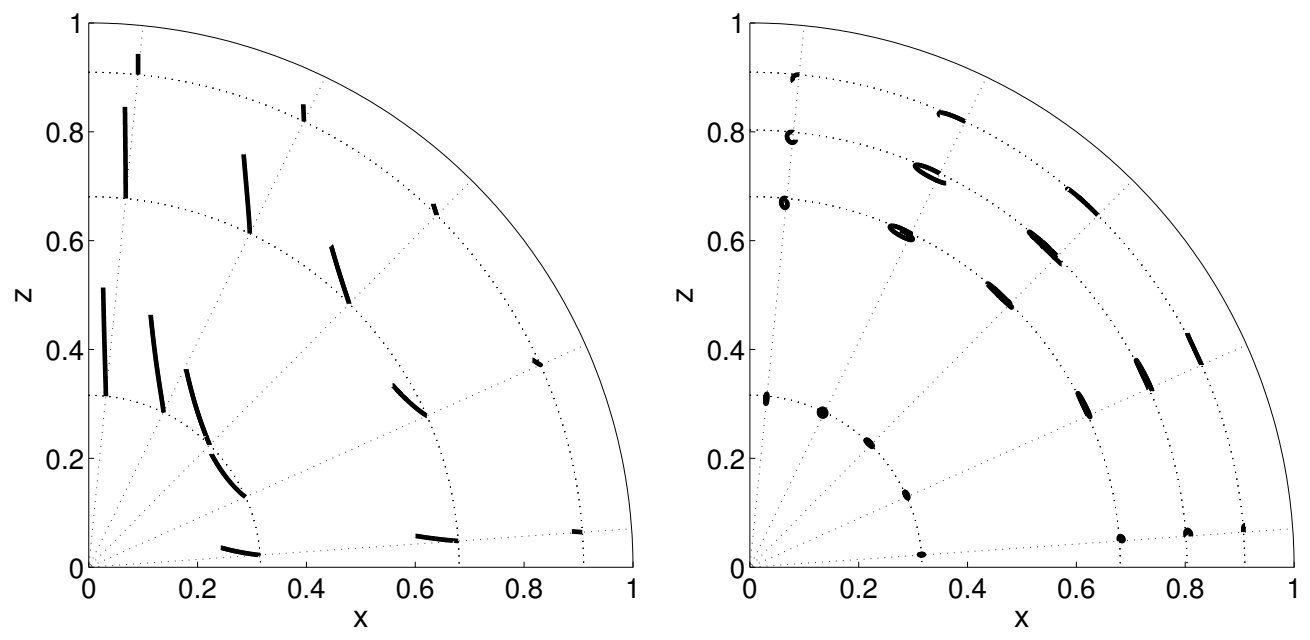

Figure 2: Trajectories within the inner core. Twenty samples are introduced at the inner core boundary at different colatitude, $\theta=5,25,45,65$ and 85 degrees, and different times, $\tilde{t}=0.1,0.5,0.7$, and 0.9. (a) model with no stratification $\left(B^{*}=0\right)$, the time $\tilde{t}=0.7$ is omitted for clarity. (b) model with stratification $\left(B^{*}=-10^{6}\right)$. 
stratification allows the flow to develop into the whole inner core. Continuous relaxation of the dynamic topography results in a flow from the equator to the poles with a quadripolar pattern. The average strain rate is rather small $\dot{\epsilon} \approx 3 \times 10^{-18} \mathrm{~s}^{-1} \approx 0.1 \mathrm{Gyr}^{-1}$ - it takes 1 Gyr to accumulate a deformation of $10 \%$ in the material sample - and the deformation induced texture is expected to be weak (section 4), except at the center of the inner core where strain rate can reach values of $1.5 \mathrm{Gyr}^{-1}$ during the first stage of the inner core growth.

Note the strong deformation of the initially spherical iso-compositional surfaces (Fig. 1c). This motion is also apprehended by the trajectories of samples set at the ICB and transported by the flow during its later evolution as shown in Fig. 2a.

\subsection{Stratified flows, $B^{*}=-10^{6}$}

Calculations for a stratified flow $\left(B^{*}=-10^{6}\right)$ are presented in Fig. 1 (right column). As explained in section 2.3, the magnitude of $B$ increases from 0 to $B^{*}$ during the growth of the inner core. The effect of the stratification is negligible early in the inner core history, but becomes rapidly important as the inner core grows and $B$ increases. The stratification tends to prevent radial motion because deformation of isodensity surfaces induces restoring buoyancy forces. As the stratification strengthens, the flow is forced to follow isodensity surfaces and becomes quasi-horizontal. The radial penetration is strongly reduced and the flow is progressively confined in a thin shear layer below the ICB, of thickness $\delta$. An important consequence of the localization of the flow is that the magnitude of the associated strain rate becomes much larger, as can be seen in Fig. 1e. 


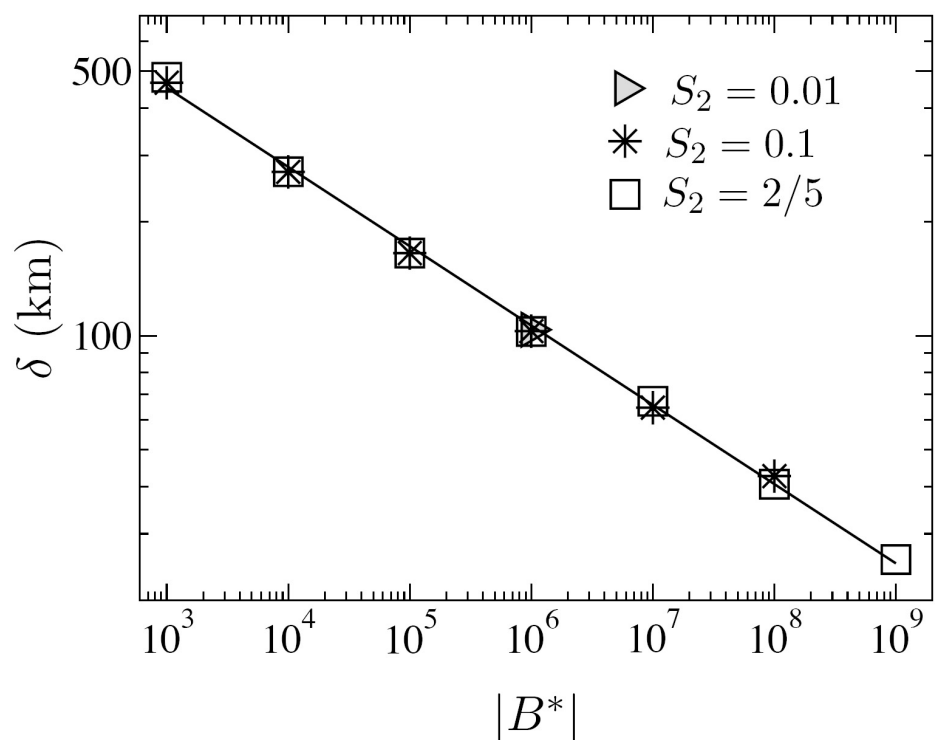

Figure 3: Evolution of the thickness $\delta$ of the uppermost stratified layer with the dimensionless buoyancy number $B^{*}$ for different values of differential growth parameter $S_{2}$ (Eq. 5). 
Momentum is transmitted deeper in the inner core by viscous entrainment, and the flow takes the form of a vertical series of elongated cells with vorticity of alternating sign. The specific geometry of the flow results from the competing effects of the stratification, which inhibits vertical motion, and of the symmetry of the forcing, which implies that the horizontal velocity must vanish in the equatorial plane and on the N-S axis. In the vicinity of the equatorial plane and of the N-S axis, the flow is deflected and forced to be locally vertical. Stratification limits this vertical flow which is forced to rotate further until it becomes horizontal again (the deformation of the isodensity surfaces acts as a localized source of vorticity). The thickness of the resulting cells depends on the magnitude of the local stratification. The associated strain rate decreases rapidly with depth and is mainly concentrated in between the two first shallower cells.

Fig. 2b shows the trajectories of different particles introduced at the surface of the inner core at different time during its growth. The trajectories are quasi horizontal below the ICB, with a maximum of amplitude at mid latitude. Deeper within the inner core, the particles follow the return flow associated with the viscous counter cell. At polar and equatorial latitudes, as well as in the deepest part of the inner core, the radial and horizontal displacement are equivalent and the particles follow almost circular trajectories.

\subsection{Scaling laws}

Simulations with different values of $S_{2}$ and $B^{*}$ have been performed. The magnitude of the flow velocity is found to be proportional to $S_{2}$ but, perhaps

surprisingly, the dimensionless depth $\tilde{\delta}$ of the alternating layers does not depend on $S_{2}$ when $S_{2}<1$. As shown in Fig. 3, the thickness of the layer 
varies with $B$ only and we find $\tilde{\delta} \approx 1.6|B|^{0.20 \pm 0.01}$ from a fit for $3<\log |B|<$ 9.

This scaling can be explained as follows. The thickness of the first cell corresponds to the depth at which the buoyancy forces resulting from the deformation of isodensity surfaces balance the viscous forces, by the mean of pressure, and prevent any further vertical motion. The uppermost cell has a dimensionless horizontal elongation equal to 1 and a dimensionless thickness $\tilde{\delta}$. From the dimensionless incompressibility equation, the horizontal component of the velocity in the layer $\tilde{v_{\theta}}$ is $O\left(S_{2} \tilde{\delta}^{-1}\right)$ since the vertical component $\tilde{v}_{r}$ imposed by the boundary condition is of order $S_{2}\left(S_{2} u_{i c b}\right.$ in dimensional unit). The vorticity $\boldsymbol{\nabla} \times \mathbf{v}$ is then $O\left(S_{2} \tilde{\delta}^{-2}\right)$. The dimensionless vorticity equation (8) writes

$$
\boldsymbol{\nabla}^{2} \boldsymbol{\nabla} \times \mathbf{v}=B \frac{\partial \tilde{c}}{\partial \theta} \mathbf{e}_{\phi}
$$

from which a scaling for $\tilde{\delta}$ can be derived. The scaling of $\partial \tilde{c} / \partial \theta$ can be found by considering the deformation by the flow in the upper cell of a newly crystallized, initially horizontal, isocompositional surface. This isocompositional surface is progressively tilted by the flow and, at a time $\delta \tilde{t}$ after solidification, the resulting horizontal compositional gradient is of order

$$
\frac{\partial \tilde{c}}{\partial \theta} \sim\left|\frac{\partial \tilde{c}}{\partial \tilde{r}}\right| \tilde{v}_{r} \delta \tilde{t} \sim S_{2} \delta \tilde{t}
$$

since $|\partial \tilde{c} / \partial \tilde{r}|=O(1)$ and $\tilde{v}_{r}=O\left(S_{2}\right)$. It reaches a maximum in the transition zone between the two upper cells, at a depth $\tilde{\delta}$, where vorticity changes sign. If the deformation velocity is small compared to the mean growth rate of the inner core, material crystallized at the ICB is buried to a depth $\tilde{\delta}$ in a time $\delta \tilde{t}=\tilde{\delta} / \tilde{u_{\mathrm{cbb}}}=\tilde{\delta}$. The maximum horizontal compositional gradient is 
then $\partial \tilde{c} / \partial \theta=O\left(S_{2} \tilde{\delta}\right)$. Using this scaling in equation (16), we find that the thickness of the shear layer scales as

$$
\tilde{\delta} \sim|B|^{-1 / 5}
$$

which has no dependence in $S_{2}$. The scaling exponent estimated above from the numerical simulations is in very good agreement with the value $1 / 5$ predicted by the scaling analysis.

The scaling law (18) implies that the strain rate in the uppermost shear layer scales as

$$
\dot{\epsilon} \sim \frac{\tilde{u_{\theta}}}{\tilde{\delta}} \sim S_{2}|B|^{2 / 5}
$$

This shows that the the magnitude of the strain rate increases with increasing stratification, in agreement with our simulations. The $2 / 5$ power is small but the buoyancy number can easily reach values large enough to concentrate the deformation in the uppermost layer. With $B^{*}=-10^{6}, \delta \sim 100 \mathrm{~km}$ and

$\dot{\epsilon} \sim 0.015 \mathrm{Myr}^{-1}$. This corresponds to a cumulative horizontal deformation of more than $100 \%$ in a material sample during its burying below the dynamical superficial layers. Note that since $B$ is an increasing function of time, $\delta$ decreases and $\dot{\epsilon}$ increases with time.

\section{Mineralogical model}

It is generally accepted that the stable phase of pure iron at inner core conditions is hexagonal close packed (hcp) (e.g. Mao et al., 1990; Ma et al., 2004; Dewaele et al., 2006; Tateno et al., 2010). The presence of light elements in the core is said to stabilize cubic phases at high temperature (Vočadlo et al., 2003; Dubrovinsky et al., 2007; Côté et al., 2008). Kuwayama et al. 
(2008) also argued that a transition from an hcp to an fcc phase could explain the presence of the innermost inner core (Ishii and Dziewoński, 2002; Niu and Chen, 2008), although the range of composition at which this can occur is tight. For the sake of simplicity, in this paper, we will restrict ourself and assume that the inner core is composed of pure hcp-Fe.

\subsection{Plastic properties}

To this day, the determination of active deformation mechanisms in iron at core conditions remains an active field of research (Poirier and Price, 1999; Wenk et al., 2000b; Merkel et al., 2004; Miyagi et al., 2008; Liermann et al., 2009) and more experiments will be needed to properly constrain those parameters.

At room temperature, plastic deformation in hcp-Fe is controlled by a dominant $(0001)\langle\overline{1} 2 \overline{1} 0\rangle$ basal slip, with a contribution of $\{10 \overline{1} 0\}\langle\overline{1} 2 \overline{1} 0\rangle$ prismatic slip, mechanical twinning, and other minor systems (Wenk et al., 2000b; Merkel et al., 2004). At higher temperature, twinning is inhibited and pyramidal $\langle c+a\rangle,\{2 \overline{11} 2\}\langle 2 \overline{11} 3\rangle$ becomes more dominant (Miyagi et al., 2008). It allows an easier rotation of the Fe grains. In agreement with those experimental results, we assume easy basal slip and allow significant contribution of prismatic and pyramidal $\langle c+a\rangle$ slip (Table 1).

LPO in Fe polycrystals were simulated using the Los Alamos viscoplastic self-consistent (VPSC) code of Lebensohn and Tomé (1993). The VPSC model treats each grain as an inclusion in a homogeneous but anisotropic medium that has the average properties of the polycrystal. It is intermediate between the Taylor model that enforces strain compatibility and the Sachs model that is based on stress equilibrium. One to one grain interaction 


\begin{tabular}{lccc}
\hline Slip type & Plane & Direction & CRSS \\
\hline Basal & $(0001)$ & $\langle\overline{1} 2 \overline{1} 0\rangle$ & 0.5 \\
Prismatic & $\{10 \overline{1} 0\}$ & $\langle\overline{1} 2 \overline{1} 0\rangle$ & 1.0 \\
Pyramidal $\langle a\rangle$ & $\{10 \overline{1} 1\}$ & $\langle\overline{1} 2 \overline{1} 0\rangle$ & 3.0 \\
Pyramidal $\langle c+a\rangle$ & $\{2 \overline{11} 2\}$ & $<2 \overline{1} 13\rangle$ & 2.0 \\
\hline
\end{tabular}

Table 1: Slip systems and their critical resolved shear stress (CRSS) of hcp-Fe used in our simulations.

or intergranular heterogeneities are not accounted for directly, but using a mean field approach. As deformation proceeds, crystals deform and rotate to generate preferred orientation. By applying different critical resolved shear stresses (CRSS) to slip systems, the model will favour one deformation mode over another. It was already used for inner core modelling by Wenk et al. (2000a) and Buffett and Wenk (2001) and has been reliable for simulating textures of many low symmetry materials (Wenk, 1999) and hcp metals (e.g. Lebensohn and Tomé, 1993; Proust et al., 2010).

For each deformation step, we extracted the velocity gradients for markers placed inside our models (Fig. 2). Those gradients are used as inputs for simulating texture evolution of a 3000 grains Fe aggregate using VPSC. Orientations in the aggregates are characterized by an orientation distribution function (ODF). The ODF is a probability function for finding an orientation and it is normalized over the whole orientation space to unity. An aggregate with a random distribution function has a probability of one for all orientations, or one multiple of a random distribution (m.r.d.). If preferred orientation is present, some orientations have probabilities higher 
than one and others lower than one. Three dimensional ODF are not easy to visualize. Here, we will display pole figures of the $\langle a\rangle$ and $\langle c\rangle$ directions. In order to match the meridional representation of the flow, we place the $z$-direction (axis of rotation of the Earth) at the top of the figure, the $x$ axis (cylindrical radial direction) at the right of the figure, and the $y$-axis (azimuthal direction) at the center.

\subsection{Initial texture}

Simulations were run with both a random initial texture and a solidificationinduced initial texture. It seems quite likely that a texture can be frozen-in at the ICB during solidification (Bergman, 1997; Brito et al., 2002), in particular if the inner core grows dendritically, as predicted by theoretical analysis (Fearn et al., 1981; Shimizu et al., 2005; Deguen et al., 2007). Dendritic crystallization of hcp materials typically leads to crystallographic preferred orientation with the basal planes of the crystals parallel to the gradient of temperature (e.g. Bergman et al., 2000, 2003). Therefore, we created distributions such as those in Fig. 4 to be used as a pretexture induced by crystallization, where $\langle a\rangle$ and $\langle c\rangle$ axes are preferentially aligned perpendicular and within the plane of the ICB, respectively.

\subsection{Time evolution of texturing}

Fig. 5 shows an example of outputs of the dynamical model with $B^{*}=$ $-10^{6}$, for a polycrystalline aggregate introduced at time $\tilde{t}=0.5$, colatitude $\theta=45^{\circ}$, and radius 0.96 . Our calculations assume an incompressible fluid and thus $\sum_{i} \dot{\epsilon}_{i i}=0$. For this particular tracer, deformation is dominated by the $x x$ and $z z$ components of the deformation tensor with $\dot{\epsilon}_{x x} \approx-\dot{\epsilon}_{z z}$. 


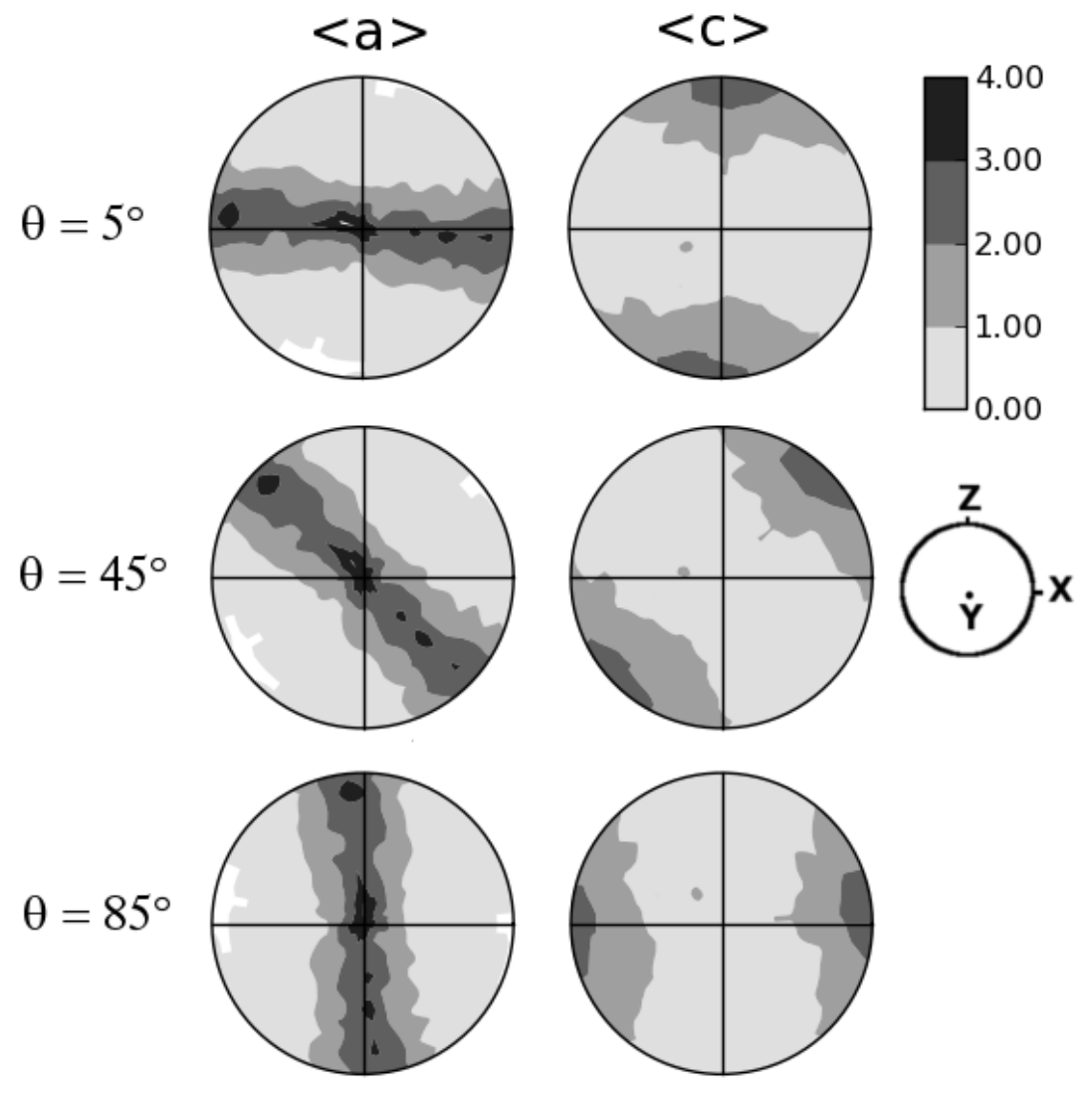

Figure 4: Pretexture due to solidification at the ICB, expressed using pole figures of the $\langle a\rangle$ and $\langle c\rangle$ directions, for colatitudes of 5,45 , and $85^{\circ}$. Linear scale, equal-area projection, contours in multiples of a random distribution (m.r.d.). 

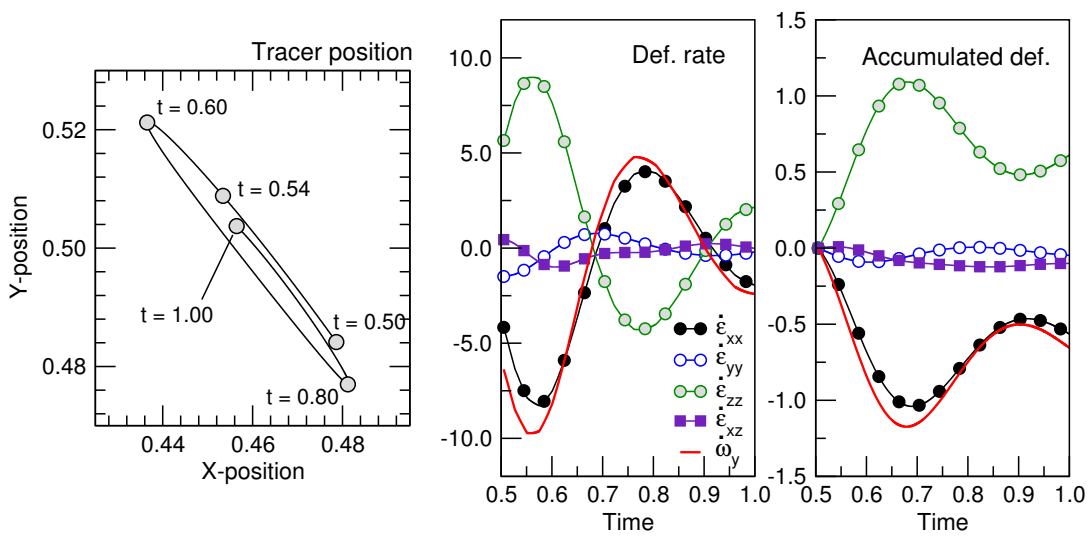

Figure 5: Trajectory, components of the strain rate tensor $\left(\dot{\epsilon}_{x x}, \dot{\epsilon}_{y y}, \dot{\epsilon}_{z z}, \dot{\epsilon}_{x y}\right)$, rigid body rotation $\left(\dot{\omega}_{y}\right)$, and accumulated deformation $\left(\epsilon_{x x}, \epsilon_{y y}, \epsilon_{z z}, \epsilon_{x y}\right)$ and rotation $\left(\omega_{y}\right)$ vs. time for a particle introduced at time $\tilde{t}=0.5$, colatitude $45^{\circ}$, and radius 0.96 .

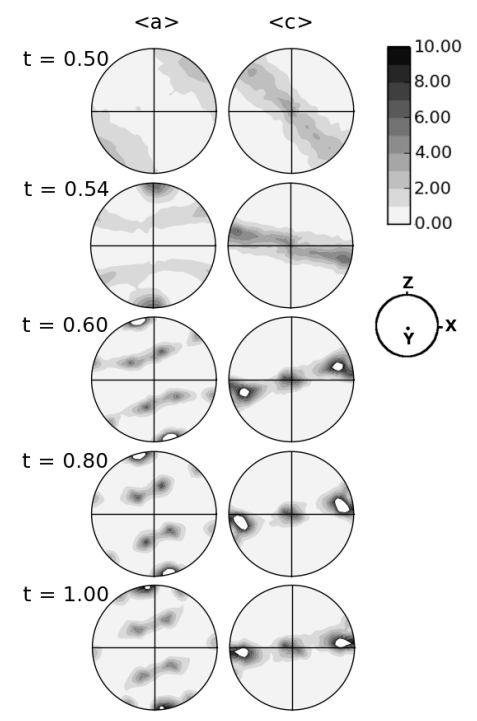

Figure 6: Pole figures of the $\langle a\rangle$ and $\langle c\rangle$ directions illustrating the LPO simulated for an hcp-Fe aggregate introduced in the inner core time $\tilde{t}=0.5$, colatitude $45^{\circ}$, and radius 0.96 (Fig. 5), assuming a crystallization texture as in Fig. 4. Contour scale in m.r.d. White color indicates regions where the pole density is larger than in the color scale. 
This corresponds to simple shear applied at $45^{\circ}$ with respect to the Earth reference frame, in the direction of the flow (Fig. 5).

The rigid body rotation of the aggregate is given by $\omega_{y}$. This component is important in magnitude and induces a rotation of the aggregate around the azimuthal axis that affects the orientation of the texture.

The corresponding texture evolution for an hcp-Fe aggregate with an initial solidification texture (Fig. 4) is shown in Fig. 6. Texture develops very quickly when the aggregate is in the upper layer and migrates toward the pole. At time $\tilde{t}=0.6$, texture is fully developed, with the $a$ and $c$-axes of the aggregate mainly distributed along directions parallel and perpendicular to the N-S direction, respectively.

\section{Evolution of lattice preferred orientation during inner core growth}

\subsection{Neutral stratification, $B^{*}=0$}

In this case, we do not account for stratification, and flow can develop in the whole inner core (section 3.1, Fig. 2a). At the center of the inner core, the deformation rate is large enough to modify the orientation of the aggregate at the beginning of the inner core formation. Later on, and consequently further out in the inner core, deformation becomes weaker and is not sufficient to induce any texture development.

With a random initial texture, LPO is significant only at the center of the inner core (Fig. 7a, aggregates introduced at $\tilde{t}=0.1$ ). The inner core itself could be seen as a sample in a pure shear deformation with a compression in the equatorial plane and an extension along the polar axis. This explains why 


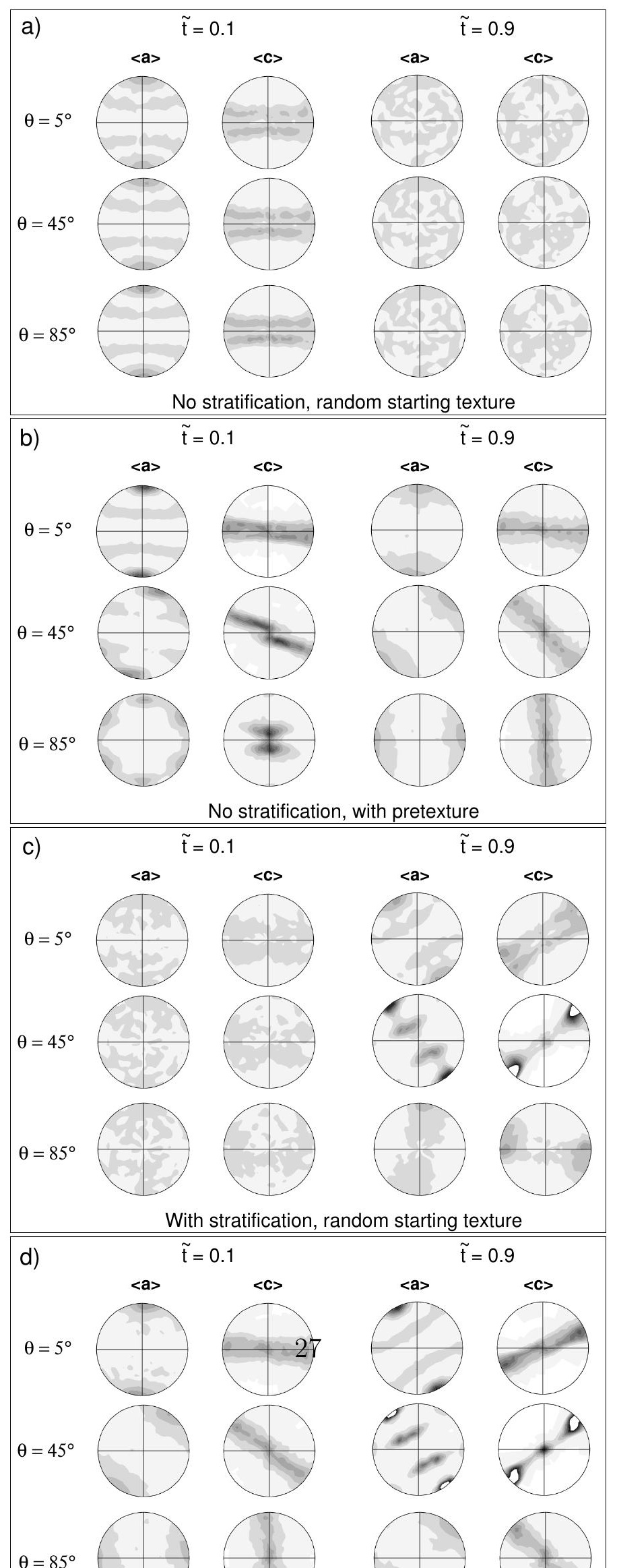


the samples crystallized at $\tilde{t}=0.1$ all show the same LPO. The distribution of $c$-axes shows a pronounced maximum $10^{\circ}$ to $15^{\circ}$ away from the equatorial plane while $a$-axes concentrate toward the poles and a secondary maximum at colatitudes of $60^{\circ}$. The $10^{\circ}$ to $15^{\circ}$ deviation of $c$-axes from the equatorial plane may be surprising at first, but is related to the activity of pyramidal $\langle c+a\rangle$ slip.

With solidification texturing as in Fig. 4, the initial textures are only weakly affected by the flow, as can be seen in Fig. 7b. The solidification texture is preserved in the outer part of the inner core (aggregates introduced at $\tilde{t}=0.9$ in Fig. 7b), and only aggregates in the central part of the inner core (introduced at $\tilde{t}=0.1$ and $\tilde{t}=0.5$ in Fig. $7 \mathrm{~b}$ ) have been significantly reworked. Close to the N-S axis, plastic deformation is compatible with the initial LPO and reinforce the alignment of the $a$-axes with the N-S axis (e.g. $\left.\tilde{t}=0.1, \theta=5^{\circ}\right)$.

\subsection{Stratified flow, $B^{*}=-10^{6}$}

For a stratified flow, and assuming random initial textures, aggregates tend to develop strong textures in the outer part of the inner core (Fig. 7c) where plastic deformation is maximum (Fig. 1e). Near the center $(r<0.5$, aggregates introduced at $\tilde{t}=0.1$, LPO is weak and the signature of plastic deformation cannot be detected. This can be attributed to the small value of the buoyancy number and the small corresponding strain rate (Eq. 19) early in inner core history. As the inner core grows and stratification strengthens, deformation is gradually localized in the upper inner core where LPO is acquired by simple shear in a relatively short time scale. There is no significant deformation in the deeper inner core (Fig. 1e), and the deep texture is 
preserved.

In the uppermost shear layer at mid latitude, the $c$-axes tend to orient along the radial direction, perpendicular to the shear plane. The $a$-axes are oriented preferentially in the horizontal plane with a primary orientation in the direction of the shear and a secondary direction at $60^{\circ}$ off the meridional plane. In the deep inner core, rigid body rotation and simple shear change these orientations, but by less than $20^{\circ}$.

In the equatorial disk and the polar cone, Fe aggregates exhibit a weak texture: the vertical migration of material in these region is associated with pure shear but is much smaller. The pure shear deformation would tend to align the $c$-axes with the major compression direction, and $a$-axes in the extension direction. From the scaling laws deduced in section 3.3, we can show that the deformation associated with the pure shear is a factor $\tilde{\delta}$ smaller than the simple shear at mid latitude. In those two regions, rigid body rotation of the aggregates plays an important role and rotates the deformation texture around the y-axis (clockwise in the equatorial disk and the opposite and the polar region).

With solidification texturing, the initial texture is preserved in the central part of the inner core (Fig. $7 \mathrm{~d}$, aggregates introduced at $\tilde{t}=0.1$ ). Along the axis of rotation (Fig. $7 \mathrm{~d}, \theta=5^{\circ}$ ) and the equatorial plane (Fig. $7 \mathrm{~d}$, $\theta=85^{\circ}$ ), the solidification texture is rotated by the rigid body rotation of the sample, as shown in Fig. 2b. In the superficial shear layer, the texture is heavily transformed to textures resembling that of Fig. 7c, where a random initial texture was assumed. 


\section{Predicted elastic anisotropy of the inner core}

\subsection{Elastic properties of hcp-Fe at inner core conditions}

Calculation of seismic wave velocities inside crystal aggregates requires the knowledge of single crystal elastic moduli. The elastic properties of iron are difficult to evaluate experimentally at inner core conditions (e.g. Mao et al., 1998; Fiquet et al., 2001; Merkel et al., 2005; Antonangeli et al., 2006; Badro et al., 2007; Mao et al., 2008) while they remain a challenge for the most advanced ab initio calculations (e.g. Stixrude and Cohen, 1995; Laio et al., 2000; Steinle-Neumann et al., 2001; Gannarelli et al., 2003, 2005; Vočadlo et al., 2009; Sha and Cohen, 2010; Chen et al., 2011). From those experiments and calculations, the anisotropy of hcp iron remains unclear. Low temperature, high pressure calculations (Stixrude and Cohen, 1995; Laio et al., 2000; Steinle-Neumann et al., 2001; Gannarelli et al., 2003, 2005; Vočadlo et al., 2009; Sha and Cohen, 2010; Chen et al., 2011) suggest that Pwave propagation is faster along the $c$-axis than along the $a$-axis, in agreement with low pressure/low temperature hcp analogs. Experimental determination of the elastic constants suggest a fast direction lying at an intermediate angle between the $a$ and $c$-axes (Mao et al., 1998; Merkel et al., 2005), but the technique that was used includes serious artifacts related to stress heterogeneities induced by plastic deformation (Antonangeli et al., 2006; Merkel et al., 2009). At inner core pressures and temperatures, Steinle-Neumann et al. (2001) found a reversal of anisotropy, with the $a$-axis faster than the $c$-axis but the validity of their calculation has been questioned (Gannarelli

et al., 2003, 2005). Yet recent ab initio calculations at inner core pressure and temperature by Vočadlo et al. (2009), Sha and Cohen (2010), and Chen 


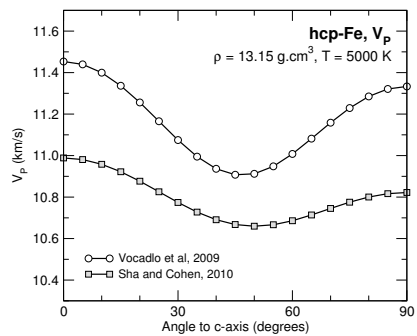

Figure 8: P-wave velocity in hcp-iron at $5000 \mathrm{~K}$ and core density according to the $a b$ initio calculations of Vočadlo et al. (2009) and Sha and Cohen (2010) as a function of propagation direction with respect to the $c$-axis. Results of Sha and Cohen (2010) have been interpolated to a density of $\rho=13.15 \mathrm{~g} . \mathrm{cm}^{-3}$ and temperature $T=5000 \mathrm{~K}$.

\begin{tabular}{cccccc}
\hline$c_{11}$ & $c_{33}$ & $c_{12}$ & $c_{23}$ & $c_{44}$ & $\rho$ \\
1689 & 1725 & 1186 & 990 & 216 & 13.154 \\
\hline
\end{tabular}

Table 2: Interpolated isothermal elastic constants (in GPa) and density (in g.m ${ }^{-3}$ ) for hcp-Fe at $5000 \mathrm{~K}$ according to Vočadlo et al. (2009). $c_{66}=\left(c_{11}-c_{12}\right) / 2$

et al. (2011) again suggest that anisotropy in iron changes at high temperature. Results of the calculations differ, both for the average $P$-wave velocity and the amplitude of anisotropy (Fig. 8). However, those calculations find that $P$-waves travel faster along the $c$ than the $a$ axis, with a pronounced minimum $45^{\circ}$ away from $c$. Here, we will use the set of elastic moduli of Vočadlo et al. (2009) (Table 2). Calculations using the elastic moduli of Sha and Cohen (2010) or Chen et al. (2011) would not change the style of anisotropy, but only the absolute $P$-waves velocities and the amplitude of anisotropy. Also note that all recent calculations find a pronounced $P$-wave velocity minimum $45^{\circ}$ away from $c$. This implies that discussing anisotropy in terms of velocities along $a$ and $c$-axes only is not appropriate. 

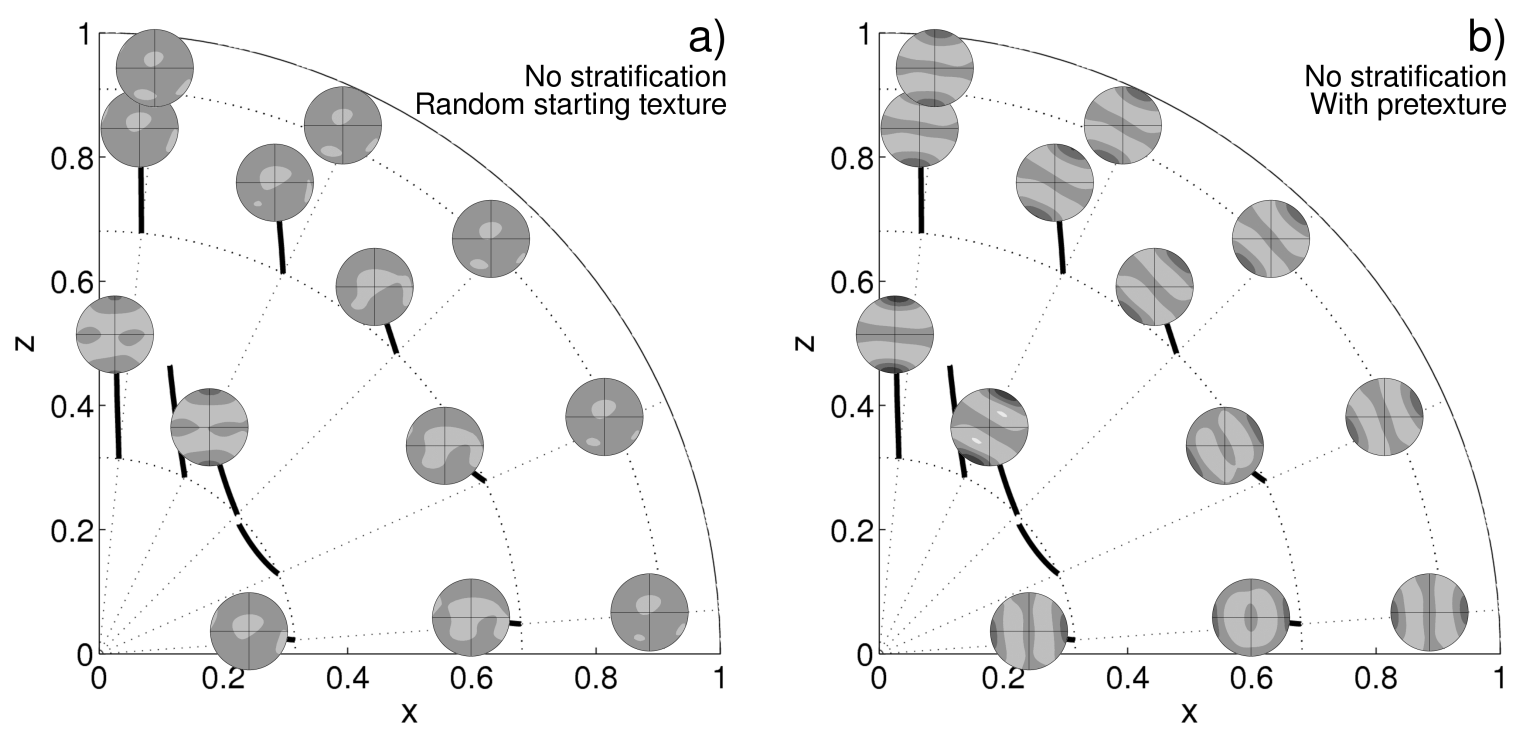

b)

a)
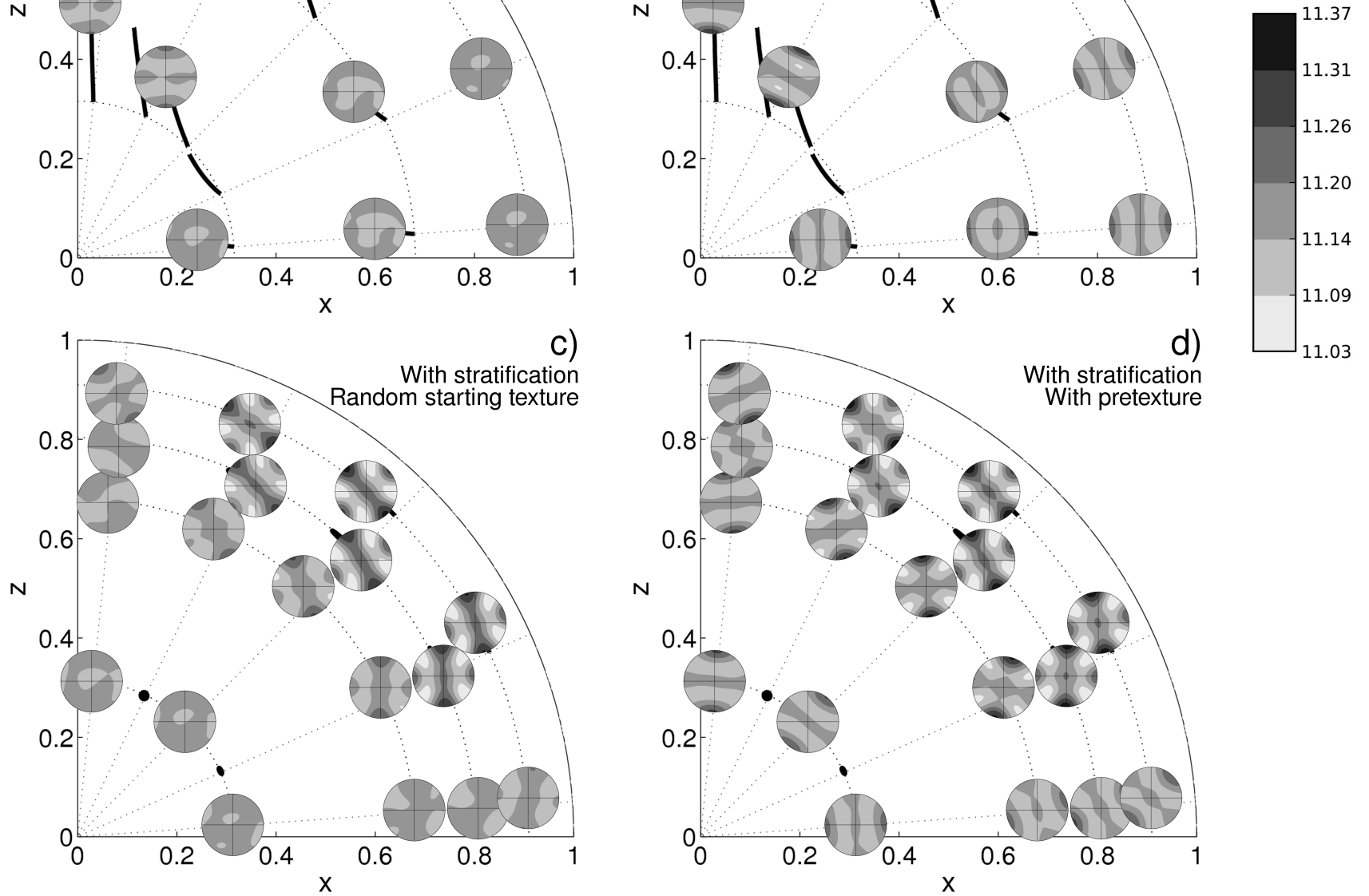

Figure 9: Present day $\mathrm{P}$ wave velocity anisotropy inside the inner core for a model with no stratification $(B=0)$, with stratification $\left(B=-10^{6}\right)$, with a random solidification texture, or with initial solidification texture. Velocity scale are in $\mathrm{km} / \mathrm{s}$. Each contour corresponds to a $0.5 \%$ change in velocity. 


\subsection{Model with no stratification and a random initial texture}

Fig. 9a shows the present day P-wave velocities inside the inner core obtained for a model with no stratification and a random initial texture. This model exhibits a cylindrical anisotropy with fast velocity along the NS axis

in the deep $(r \lesssim 0.5)$ inner core, and isotropy in the upper half of the inner core. The overall pattern of anisotropy seems reasonably consistent with seismological observations (deep cylindrical anisotropy and isotropic layer) but the isotropic region is too thick and the global anisotropy is significantly weaker than observed. The local P-wave anisotropy is at most $\sim 1 \%$, which implies that global travel time anisotropy would be significantly less than $1 \%$. This is far from the 3\% anomaly observed by seismologists (Souriau, 2003). In addition, the slowest direction in this model is not in the equatorial plane but at about $45^{\circ}$ from the N-S axis.

\subsection{Model with no stratification and solidification texturing}

Solidification texturing can produce strong anomalies at the top of the inner core. In this model, local P-wave anisotropy can be seen throughout the inner core, with amplitudes reaching about 1.5\% (Fig. 9b). In most of the inner core, the calculated direction of fast propagation is mostly radial, with a secondary maximum in the plane perpendicular to the radial direction. This configuration poorly explains the seismological data since ray paths integrations would not show significant variations of travel time with the ray angle. 


\subsection{Model with stratification and a random initial texture}

Stratification inhibits radial flows, concentrates the deformation in a superficial horizontal layer and produces intense simple shear deformation that can generate strong LPO and seismic anisotropy. Fig. 9a shows the present day $\mathrm{P}$-wave velocity distribution inside the inner core for a model with $B^{*}=-10^{6}$ and a random initial texture.

With stratification $\left(B^{*}=-10^{6}\right)$, the anisotropy is large $(3 \%)$ but is mostly localized in the outer part of the inner core, away from the equator and the pole (Fig. 9c). The directions of fast propagation are mainly oriented along the direction of shear, parallel to the ICB, with a slight rotation as the material plunges deeper into the inner core. This rotation induces a shift of the direction of fast propagation from the local horizontal towards the N-S direction (e.g. $r \approx 0.7$ ). Note the quadrangular shape of the anisotropy figure which is the reminiscence of the $\mathrm{P}$-wave velocity minimum at $45^{\circ}$ away from $a$ and $c$ in the single crystal (Fig. 8). The anisotropy is weak in the deep inner core, in disagreement with seismic observations.

The strong radial anisotropy in the uppermost layer would be difficult to detect. Constraints on the seismic properties of the uppermost inner core come mostly from the comparison of almost horizontal rays with different orientations. This is insensitive to radial anisotropy, which implies that the prediction of radial anisotropy is not incompatible with the apparent isotropy of the uppermost inner core. It would probably be difficult to compare horizontal and radial traveltimes in this layer. 


\subsection{Model with stratification and solidification texturing}

This model results in a layered inner core with two layers of distinct anisotropy. Similarly to the previous model, there is a strong anisotropy in the outer part of the inner core, away from the equator and the pole (Fig. $9 \mathrm{~b})$. In this region, the crystallization texture is erased and the observed anisotropy is the result of the intense shear flow induced by stratification. Below $r<0.5$, LPO associated with the solidification process is preserved. Along the equator and the N-S axis, the anisotropy induced by solidification is slightly rotated. Indeed, in those regions, aggregate are subjected to significant rigid-body rotation but little plastic deformation (see Fig. 2).

\section{Discussion and conclusions}

We have successfully coupled a model of inner core dynamics with a viscoplastic deformation model to compute LPO of hcp-Fe in the inner core and predict the resulting seismological anisotropy. Although the procedure we have developed is quite general, each step involves additional hypothesis, and consequently less robustness. The final result is function of a number of assumptions regarding the thermo-chemical state of the inner core, the stable phase of iron at inner core condition, its rheology, and its elastic properties. The choices we have made are consistent with current beliefs and the most recent experimental and numerical studies, but there is still no consensus on these questions.

Despite these difficulties, a number of general results emerge from our work:

i) Flow and stress field calculated by Yoshida et al. (1996) are applicable 
to the inner core only if $|B| \lesssim 10^{2}$ (see Fig. 3). Since the order of magnitude of the stratification is probably correctly estimated, $B$ is mostly sensitive to the viscosity. Having $\left|B^{*}\right|<10^{2}$ requires $\eta>10^{22}$ Pa.s (Eq. 12), at the very high end of published estimates (Yoshida et al., 1996). If the inner core is stably stratified, the forcing proposed by Yoshida et al. (1996) - viscous relaxation of a disequilibrium topography sustained by heterogeneous solidification - is still relevant, but the flow is significantly altered. Stratification inhibits vertical motion and deformation is localized in a shallow shear layer. Perhaps surprisingly, the effect of a stable stratification is to increase the magnitude of the strain rate and the efficiency of deformation induced texturation. Deformation in this shear layer erases any solidification texturing, and results in a strong, predominantly radial, anisotropy. This layer would appear isotropic to body wave seismology, but might be detectable in normal mode data.

ii) An interesting consequence of the increase of $|B|$ during inner core history is that there is a gradual, but significant, change in the flow geometry and in the strain rate magnitude. As illustrated in Figs. 7 and 9, this can result in a layered inner core, with the deepest part of the inner core only very weakly affected by deformation, and the upper layer with an heavily reworked texture. Our simulations of texture development show that, in particular, a solidification texture can be preserved in the deep inner core. The relative thickness of the two layers depend on the value of the buoyancy number $B^{*}$. Larger value of $B^{*}$ would result in a smaller region of fossilized solidification texture.

iii) It is important to constrain whether inner core anisotropy results pri- 
marily from currently active processes, or from past texturation processes. The distinction is important in particular because true polar wander of the inner core is plausible at long time scales. Therefore, the orientation of a fossil anisotropy may not be conserved. In our model of inner core layering, texturation in the outermost inner core results from intense plastic deformation, and this process is fast $(\sim 10 \mathrm{Myr})$, but anisotropy in the deep inner core is mostly fossil. If inner core true polar wander is indeed significant, the initial orientation of the deep inner core anisotropy could have been rotated in a way that would be difficult to predict. This is not necessarily incompatible with seismic observations (e.g. Niu and Chen, 2008) that suggest a different orientation of anisotropy in the innermost inner core.

iv) Chemical stratification combined with crystallization texture is a powerful mechanism for generating anisotropy in the inner core. This results in a layered inner core with distinct seismic properties (Fig. 9d). The amplitude of the calculated anisotropy is of the same order of magnitude that seismic observations, with a complex substructure. Because of this complex substructure, a comparison with first order seismic anisotropy observations is difficult. This will be addressed in a forthcoming study.

v) Finally, our model can be generalized to any arbitrary inner core growth pattern. In particular, the hemispherical growth pattern as predicted by the experiments of Sumita and Olson $(1999,2002)$ and the numerical simulations of Aubert et al. (2008) is an interesting candidate for explaining the seismic hemispherical dichotomy of the inner core (Tanaka and Hamaguchi, 1997; Garcia and Souriau, 2000; Deuss et al., 2010). This should be investigated in the future. 
Acknowledgments. All computations presented here were performed at the Service Commun de Calcul Intensif de l'Observatoire de Grenoble. We thank the undergraduate students Jonathan Serafini and Charlotte Regnier who participated to the study. R. Deguen was partly supported by NSF grant EAR 0909622. S. Merkel was supported by ANR program DiUP, N. ANR-07JCJC-0136. The study was granted by the program SEDIT of INSU/CNRS.

\section{References}

Albarède, F., 1996. Introduction to geochemical modeling. Cambridge University Press, Cambridge, UK.

Alboussière, T., Deguen, R., Melzani, M., 2010. Melting induced stratification above the Earth's inner core due to convective translation. Nature 466, 744-747.

Alfè, D., Gillan, M. J., Price, G. D., 2002. Ab initio chemical potentials of solid and liquid solutions and the chemistry of the Earth's core. J. Chem. Phys. 116, 7127-7136.

Allègre, C. J., Poirier, J.-P., Humler, E., Hofmann, A. W., 1995. The chemical composition of the Earth. Earth Planet. Sci. Lett. 134, 515-526.

Antonangeli, D., Merkel, S., Farber, D. L., 2006. Elastic anisotropy in hcp metals at high pressure and the sound wave anisotropy of the Earth's inner core. Geophys. Res. Lett. 33, L24303.

Aubert, J., Amit, H., Hulot, G., Olson, P., 2008. Thermochemical flows 
couple the Earth's inner core growth to mantle heterogeneity. Nature 454, 758-761.

Aurnou, J., Heimpel, M., Allen, L., King, E., Wicht, J., 2008. Convective heat transfer and the pattern of thermal emission on the gas giants. Geophys. J. Int. 173, 793-801.

Badro, J., Fiquet, G., Guyot, F., Gregoryanz, E., Occelli, F., Antonangeli, D., D'Astuto, M., 2007. Effect of light elements on the sound velocities in solid iron: Implications for the composition of Earth's core. Earth Planet. Sci. Lett. 254, 233-238.

Bergman, M. I., 1997. Measurements of elastic anisotropy due to solidification texturing and the implications for the Earth's inner core. Nature 389, 6063.

Bergman, M. I., Agrawal, S., Carter, M., Macleod-Silberstein, M., 2003. Transverse solidification textures in hexagonal close-packed alloys. J. Crystal Growth 255, 204-211.

Bergman, M. I., Giersch, L., Hinczewski, M., Izzo, V., 2000. Elastic and attenuation anisotropy in directionally solidified (hcp) zinc, and the seismic anisotropy in the Earth's inner core. Phys. Earth Planet. Inter. 117, 139151.

Brito, D., Elbert, D., Olson, P., 2002. Experimental crystallization of gallium: ultrasonic measurements of elastic anisotropy and implications for the inner core. Phys. Earth Planet. Inter. 129, 325-346. 
Buffett, B. A., 1997. Geodynamic estimates of the viscosity of the Earth's inner core. Nature 388, 571-573.

Buffett, B. A., 2009. Onset and orientation of convection in the inner core. Geophys. J. Int. 179, 711-719.

Buffett, B. A., Bloxham, J., 2000. Deformation of Earth's inner core by electromagnetic forces. Geophys. Res. Lett. 27, 4001-4004.

Buffett, B. A., Huppert, H. E., Lister, J. R., Woods, A. W., 1992. Analytical model for solidification of the Earth's core. Nature 356, 329-331.

Buffett, B. A., Wenk, H.-R., 2001. Texturing of the Earth's inner core by Maxwell stresses. Nature 413, 60-63.

Busse, F. H., 1970. Thermal instabilities in rapidly rotating systems. J. Fluid Mech. 44, 441-460.

Calvet, M., Chevrot, S., Souriau, A., 2006. P-wave propagation in transversely isotropic media: II. Application to inner core anisotropy: Effects of data averaging, parametrization and a priori information. Phys. Earth Planet. Inter. 156, 21-40.

Cardin, P., Olson, P., 1994. Chaotic thermal convection in a rapidly rotating spherical shell: consequences for flow in the outer core. Phys. Earth Planet. Inter. 82, 235-259.

Chen, X.-R., Zeng, Z.-Y., Liu, Z.-L., Cai, L.-C., Jing, F.-Q., 2011. Elastic anisotropy of $\epsilon$-fe under conditions at the earth's inner core. Phys. Rev. B 83, 132102. 
Côté, A. S., Vočadlo, L., Brodholt, J. P., 2008. Light elements in the core: Effects of impurities on the phase diagram of iron. Geophys. Res. Lett. 35, L05306.

Crank, J., 1984. Free and moving boundary problems. Clarendon Press, Oxford, UK.

Deguen, R., Alboussière, T., Brito, D., 2007. On the presence and structure of a mush at the inner core boundary of the Earth. Phys. Earth Planet. Inter. 274, 1887-1891.

Deguen, R., Cardin, P., 2009. Tectonic history of the Earth's inner core preserved in its seismic structure. Nat. Geosci. 2, 419-422.

Deguen, R., Cardin, P., 2011. Thermo-chemical convection in the Earth's inner core. Geophys. J. Int., submitted.

Deuss, A., Irving, J., Woodhouse, J., 2010. Regional variation of inner core anisotropy from seismic normal mode observations. Science 328, 1018.

Dewaele, A., Loubeyre, P., Occelli, F., Mezouar, M., Dorogokupets, P. I., Torrent, M., 2006. Quasihydrostatic equation of state of iron above 2 mbar. Phys. Rev. Lett. 97, 215504.

Dormy, E., Soward, A. M., Jones, C. A., Jault, D., Cardin, P., 2004. The onset of thermal convection in rotating spherical shells. J. Fluid Mech. 501, 43-70.

Dubrovinsky, L., Dubrovinskaia, N., Narygina, O., Kantor, I., Kuznetzov, A., Prakapenka, V. B., Vitos, L., Johansson, B., Mikhaylushkin, A. S., Simak, 
S. I., Abrikosov, I. A., 2007. Body-Centered Cubic Iron-Nickel Alloy in Earth's Core. Science 316, 1880-1883.

Fearn, D., Loper, D., Roberts, P., 1981. Structure of the Earth's inner core. Nature 292, 232-233.

Fiquet, G., Badro, J., Guyot, F., Requardt, H., Krisch, M., 2001. Sound velocities in iron to 100 gigapascals. Science 291, 468-471.

Gannarelli, C. M. S., Alfè, D., Gillan, M. J., 2003. The particle-in-cell model for ab initio thermodynamics: implications for the elastic anisotropy of the Earth's inner core. Phys. Earth Planet. Inter. 139, 243-253.

Gannarelli, C. M. S., Alfè, D., Gillan, M. J., 2005. The axial ratio of hcp iron at the conditions of the Earth's inner core. Phys. Earth Planet. Inter. $152,67-77$.

Garcia, R., 2002. Constraints on upper inner-core structure from waveform inversion of core phases. Geophys. J. Int. 150, 651-664.

Garcia, R., Souriau, A., 2000. Inner core anisotropy and heterogeneity level. Geophys. Res. Lett. 27, 3121-3124.

Ishii, M., Dziewoński, A. M., 2002. The innermost inner core of the Earth: Evidence for a change in anisotropic behavior at the radius of about 300 km. Proc. Natl. Acad. Sci. 99, 14026-14030.

Jeanloz, R., Wenk, H., 1988. Convection and anisotropy of the inner core. Geophys. Res. Lett. 15, 72-75. 
Karato, S., 1993. Inner core anisotropy due to the magnetic field-induced preferred orientation of iron. Science 262, 1708-1711.

Karato, S., 1999. Seismic anisotropy of the Earth's inner core resulting from flow induced by Maxwell stresses. Nature 402, 871-873.

Kuwayama, Y., Hirose, K., Sata, N., Ohishi, Y., 2008. Phase relations of iron and iron-nickel alloys up to $300 \mathrm{GPa}$ : Implications for composition and structure of the earth's inner core. Earth Planet. Sci. Lett. 273 (3-4), 379-385.

Labrosse, S., Poirier, J.-P., Le Mouël, J.-L., 2001. The age of the inner core. Earth Planet. Sci. Lett. 190, 111-123.

Laio, A., Bernard, S., Chiarotti, G. L., Scandolo, S., Tosatti, E., 2000. Physics of iron at Earth's core conditions. Science 287, 1027-1030.

Lebensohn, R. A., Tomé, C. N., 1993. Self-consistent anisotropic approach for the simulation of plastic deformation and texture development of polycrystals: application to zirconium alloys. Acta Metall. Mater. 41, 2611-2624.

Liermann, H.-P., Merkel, S., Miyagi, L., Wenk, H.-R., Shen, G., Cynn, H., Evans, W. J., 2009. New experimental method for in situ determination of material textures at simultaneous high-pressure and temperature by means of radial diffraction in the diamond anvil cell. Rev. Sci. Instrum. 80, 104501.

Loper, D. E., 1983. Structure of the inner core boundary. Geophys. and Astrophys. Fluid Dyn. 25, 139-155. 
Ma, Y., Somayazulu, M., Shen, G., Mao, H., Shu, J., Hemley, R., 2004. In situ X-ray diffraction studies of iron to Earth-core conditions. Phys. Earth Planet. Inter. 143-144, 455-467.

Mao, H.-K., Shu, J., Shen, G., Hemley, R. J., Li, B., Singh, A. K., 1998. Elasticity and rheology of iron above 220GPa and the nature of the Earth's inner core. Nature 396, 741-743.

Mao, H. K., Wu, Y., Chen, L. C., Shu, J. F., Jephcoat, A. P., 1990. Static compression of iron to $300 \mathrm{GPa}$ and $\mathrm{Fe}_{0.8} \mathrm{Ni}_{0.2}$ alloy to $260 \mathrm{GPa}$ : Implications for composition of the core. J. Geophys. Res. 95, 21737-21742.

Mao, W. L., Struzhkin, V. V., Baron, A. Q. R., Tsutsui, S., Tommaseo, C. E., Wenk, H.-R., Hu, M. Y., Chow, P., Sturhahn, W., Shu, J., Hemley, R. J., Heinz, D. L., Mao, H.-K., 2008. Experimental determination of the elasticity of iron at high pressure. J. Geophys. Res. 113, B09213.

Merkel, S., Shu, J., Gillet, P., Mao, H.-K., Hemley, R. J., 2005. X-ray diffraction study of the single-crystal elastic moduli of $\epsilon$-Fe up to $30 \mathrm{GPa}$. J. Geophys. Res. 110 (9), B05201.

Merkel, S., Tomé, C., Wenk, H.-R., 2009. A modeling analysis of the influence of plasticity on high pressure deformation of hcp-Co. Phys. Rev. B 79, 064110.

Merkel, S., Wenk, H., Gillet, P., Mao, H., Hemley, R. J., 2004. Deformation of polycrystalline iron up to $30 \mathrm{GPa}$ and $1000 \mathrm{~K}$. Phys. Earth Planet. Inter. 145, 239-251. 
Miyagi, L., Kunz, M., Knight, J., Nasiatka, J., Voltolini, M., Wenk, H., 2008. In situ phase transformation and deformation of iron at high pressure and temperature. J. Appl. Phys. 104, 103510.

Monnereau, M., Calvet, M., Margerin, L., Souriau, A., 2010. Lopsided growth of Earth's inner core. Science 328, 1014-1017.

Morelli, A., Dziewonski, A. M., Woodhouse, J. H., 1986. Anisotropy of the inner core inferred from PKIKP travel times. Geophys. Res. Lett. 13, 15451548.

Nimmo, F., 2007. Treatise on Geophysics. Vol. 8. Elsevier, Ch. Energetics of the Core, pp. 31-65.

Niu, F., Chen, Q., 2008. Seismic evidence for distinct anisotropy in the innermost inner core. Nat. Geosci. 1, 692-696.

Poirier, J. P., Price, G. D., 1999. Primary slip system of $\epsilon$-iron and anisotropy of the Earth's inner core. Phys. Earth Planet. Inter. 110, 147-156.

Poupinet, G., Pillet, R., Souriau, A., 1983. possible heteregeneity of the Earth's core deduced from PKIKP travel-times. Nature 305 (5931), 204206.

Proust, G., Kaschner, G. C., Beyerlein, I. J., Clausen, B., Brown, D. W., McCabe, R. J., Tom, C. N., 2010. Detwinning of high-purity zirconium: In-situ neutron diffraction experiments. Exp. Mech. 50, 125-133.

Sha, X., Cohen, R. E., 2010. Elastic isotropy of $\epsilon$-Fe under Earth's core conditions. Geophys. Res. Lett. 37, L10302. 
Shimizu, H., Poirier, J.-P., Le Mouël, J.-L., 2005. On crystallization at the inner core boundary. Phys. Earth Planet. Inter. 151, 37-51.

Song, X., Helmberger, D. V., 1995. Depth dependence of anisotropy of Earth's inner core. J. Geophys. Res. 100, 9805-9816.

Souriau, A., 2003. The seismological picture of the inner core: structure and rotation. C. R. Geosci. 335, 51-63.

Souriau, A., 2007. Treatise on Geophysics. Vol. 1. Elsevier, Ch. The Earth's Core, pp. 655-693.

Souriau, A., Romanowicz, B., 1996. Anisotropy in inner core attenuation: a new type of data to constrain the nature of the solid core. Geophys. Res. Lett. 23, 1-4.

Souriau, A., Romanowicz, B., 1997. Anisotropy in the inner core: relation between P-velocity and attenuation. Phys. Earth Planet. Inter. 101, 33-47.

Steinle-Neumann, G., Stixrude, L., Cohen, R. E., Gülseren, O., 2001. Elasticity of iron at the temperature of the Earth's inner core. Nature 413, $57-60$.

Stixrude, L., Cohen, R. E., 1995. High-pressure elasticity of iron and anisotropy of Earth's inner core. Science 267, 1972-1975.

Su, W., Dziewonski, A. M., Jeanloz, R., 1996. Planet within a planet: rotation of the inner core of Earth. Science 274, 1883-1887.

Sumita, I., Olson, P., 1999. A laboratory model for convection in Earth's core driven by a thermallyheterogeneous mantle. Science 286, 1547-1549. 
Sumita, I., Olson, P., 2002. Rotating thermal convection experiments in a hemispherical shell with heterogeneous boundary heat flux: Implications for the Earth's core. J. Geophys. Res. 107, 2169.

Sumita, I., Yoshida, S., Hamano, Y., Kumazawa, M., 1995. A model for the structural evolution of the earth's core and its relation to the observations. In: Yukutake, T. (Ed.), The Earth's central part : Its structure and dynamics. pp. 232-260.

Sumita, I., Yoshida, S., Kumazawa, M., Hamano, Y., 1996. A model for sedimentary compaction of a viscous media and its application to innercore growth. Geophys. J. Int. 124, 302-324.

Takehiro, S., 2010. Fluid motions induced by horizontally heterogeneous Joule heating in the Earth's inner core. Phys. Earth Planet. Inter. 184, $134-142$.

Tanaka, S., Hamaguchi, H., 1997. Degree one heterogeneity and hemispherical variation of anisotropy in the inner core from $\operatorname{PKP}(\mathrm{BC})-\mathrm{PKP}(\mathrm{DF})$ times. J. Geophys. Res. 102, 2925-2938.

Tateno, S., Hirose, K., Ohishi, Y., Tatsumi, Y., 2010. The structure of iron in Earth's inner core. Science 330, 359.

Tomé, C., Canova, G., Kocks, U., Christodoulou, N., Jonas, J., 1984. The relation between macroscopic and microscopic strain hardening in f.c.c. polycrystals. Acta metall. 32, $1637-1653$.

Turcotte, D. L., Schubert, G., 2002. Geodynamics. Cambridge University Press, Cambridge, UK. 
Van Orman, J. A., 2004. On the viscosity and creep mechanism of Earth's inner core. Geophys. Res. Lett. 31, L20606.

Vočadlo, L., Alfè, D., Gillan, M. J., Wood, I., Brodholt, J., Price, G. D., 2003. Possible thermal and chemical stabilization of body-centred-cubic iron in the Earth's core. Nature 424, 536-539.

Vočadlo, L., Dobson, D. P., Wood, I. G., 2009. Ab initio calculations of the elasticity of hcp-Fe as a function of temperature at inner-core pressure. Earth Planet. Sci. Lett. 288, 534-538.

Weber, P., Machetel, P., 1992. Convection within the inner-core and thermal implications. Geophys. Res. Lett. 19, 2107-2110.

Wenk, H. R., 1999. A voyage through the deformed Earth with the selfconsistent model. Model. Simul. Mater. Sci. Engin. 7, 699-722.

Wenk, H. R., Baumgardner, J. R., Lebensohn, R. A., Tomé, C. N., 2000a. A convection model to explain anisotropy of the inner core. J. Geophys. Res. $105,5663-5678$.

Wenk, H. R., Matthies, S., Hemley, R. J., Mao, H. K., Shu, J., 2000b. The plastic deformation of iron at pressures of the Earth's inner core. Nature 405, 1044-1047.

Wenk, H. R., Takeshita, T., Jeanloz, R., Johnson, G. C., 1988. Development of texture and elastic anisotropy during deformation of hcp metals. Geophys. Res. Lett. 15, 76-79. 
Woodhouse, J. H., Giardini, D., Li, X.-D., 1986. Evidence for inner core anisotropy from free oscillations. Geophys. Res. Lett. 13, 1549-1552.

Worster, M. G., 1997. Convection in mushy layers. Annu. Rev. Fluid Mech. 29, 91-122.

Yoshida, S., Sumita, I., Kumazawa, M., 1996. Growth model of the inner core coupled with the outer core dynamics and the resulting elastic anisotropy. J. Geophys. Res. 101, 28085-28104.

Yu, W., Wen, L., 2006a. Inner core attenuation anisotropy. Earth Planet. Sci. Lett. 245, 581-594.

Yu, W., Wen, L., 2006b. Seismic velocity and attenuation structures in the top $400 \mathrm{~km}$ of the Earth's inner core along equatorial paths. J. Geophys. Res. 111, B07308.

Yukutake, T., 1998. Implausibility of thermal convection in the Earth's solid inner core. Phys. Earth Planet. Inter. 108, 1-13.

Zhang, K., 1992. Spiralling columnar convection in rapidly rotating spherical fluid shells. J. Fluid Mech. 236, 535-556. 